\title{
Local Electronic Structure and Density of Edge and Facet Atoms at Rh Nanoclusters Self-Assembled on a Graphene Template
}

\author{
Alberto Cavallin, ${ }^{\dagger, \mp}$ Monica Pozzo, ${ }^{\S}$ Cristina Africh, ${ }^{\perp}$ Alessandro Baraldi, ${ }^{\dagger, \perp, *}$ Erik Vesselli, ${ }^{\dagger, \perp}$ Carlo Dri, ${ }^{\perp}$ \\ Giovanni Comelli, ${ }^{\dagger, \perp}$ Rosanna Larciprete," Paolo Lacovig, "Silvano Lizzit, " and Dario Alfè ${ }^{\S, \#}$ \\ ${ }^{\dagger}$ Physics Department and CENMAT, University of Trieste, Via Valerio 2, I-34127 Trieste, Italy, ${ }^{\ddagger}$ Institute of Condensed Matter Physics, Ecole Polytechnique Fédérale de \\ Lausanne, Station 3, CH-1015 Lausanne, Switzerland, ${ }^{\S}$ Department of Earth Sciences, Department of Physics and Astronomy, and London Centre for \\ Nanotechnology, University College London, Gower Street, London WC1E 6BT, United Kingdom, ${ }^{\perp}$ IOM-CNR Laboratorio TASC, Area Science Park, S.S. 14 Km 163.5, \\ I-34149 Trieste, Italy, "CNR-Institute for Complex Systems, Via Fosso del Cavaliere 100, 00133 Roma, Italy, " Sincrotrone Trieste S.C.p.A., Area Science Park, \\ S.S. 14 Km 163.5, I-34149 Trieste, Italy, and "IOM-CNR, DEMOCRITOS National Simulation Centre, I-34100 Trieste, Italy
}

$\mathrm{T}$ he interest in developing novel materials using building blocks with sizes of about $1 \mathrm{~nm}$ has motivated the huge effort devoted to understand the properties of nanoclusters. Several studies have clearly demonstrated that atomic aggregates in the nanometer size range, that is, formed by dozens or hundreds of atoms, present remarkably different properties with respect to their bulk crystalline counterparts. Heterogeneous catalysis, energy conversion, and magnetic data storage are among the technologically most relevant fields where supported nanoclusters are expected to have a strong impact. ${ }^{1,2}$

An important field for application of the new nanocluster-based materials is catalysis. The most striking example is the high chemical activity of small Au nanoclusters, ${ }^{3-6}$ given by highly reactive corner and edge atoms with low coordination number $C_{N}$, but even other transition metals like Pt present nanoclusters with a similar behavior. $^{7,8}$

One of the goals of nanotechnology is the manipulation of the magnetic properties in systems with reduced dimensionality, such as quantum wires, quantum dots, and nanoclusters. As an example, the use of nanoclusters for ultrahigh density magnetic recording requires pushing further away the superparamagnetic limit by enhancing the magnetic anisotropy energy. In this respect, experiments have shown that low $C_{N}$ atoms play a pivotal role. ${ }^{9,10}$

When the particle's size is reduced to only 10-20 A, two main challenges are posed: (i) the formation in a controllable and reproducible manner of large-scale replicas of these individual building blocks on a

\section{ABSTRACT}

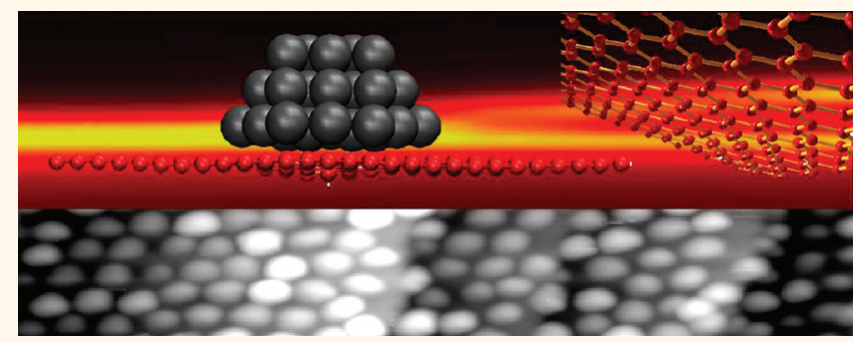

The chemical and physical properties of nanoclusters largely depend on their sizes and shapes. This is partly due to finite size effects influencing the local electronic structure of the nanocluster atoms which are located on the nanofacets and on their edges. Here we present a thorough study on graphene-supported Rh nanocluster assemblies and their geometrydependent electronic structure obtained by combining high-energy resolution core level photoelectron spectroscopy, scanning tunneling microscopy, and density functional theory. We demonstrate the possibility to finely control the morphology and the degree of structural order of Rh clusters grown in register with the template surface of graphene/Ir(111). By comparing measured and calculated core electron binding energies, we identify edge, facet, and bulk atoms of the nanoclusters. We describe how small interatomic distance changes occur while varying the nanocluster size, substantially modifying the properties of surface atoms. The properties of under-coordinated Rh atoms are discussed in view of their importance in heterogeneous catalysis and magnetism.

KEYWORDS: graphene · nanoclusters · core level photoelectron spectroscopy · scanning tunneling microscopy · density functional theory · rhodium · catalysis

suitable substrate ${ }^{11}$ and (ii) the determination and tuning of the geometric and electronic structure of the supported nanoobjects.

One of the most recent solutions to meet the first challenge is to make use of template graphene (GR) for the growth of metallic nanoclusters and for the formation of long-range-ordered superstructures. This
* Address correspondence to alessandro.baraldi@elettra.trieste.it.

Received for review November 24, 2011 and accepted March 9, 2012.

Published online March 09, 2012 $10.1021 / \mathrm{nn} 300651 \mathrm{~s}$

(c) 2012 American Chemical Society 

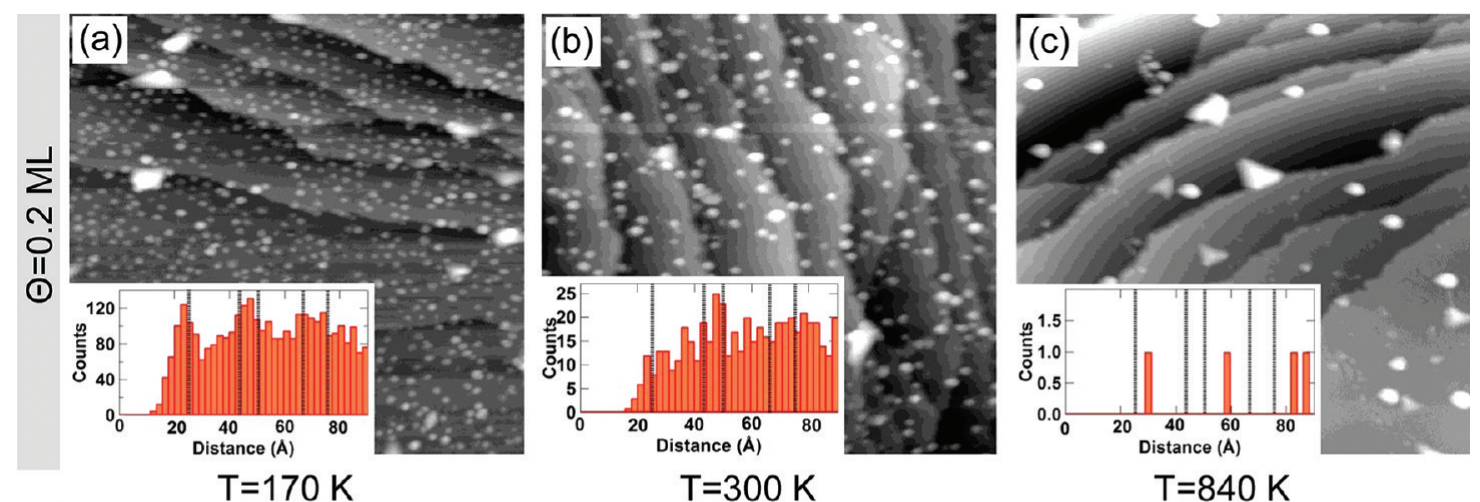

$\mathrm{T}=300 \mathrm{~K}$

$\mathrm{T}=840 \mathrm{~K}$
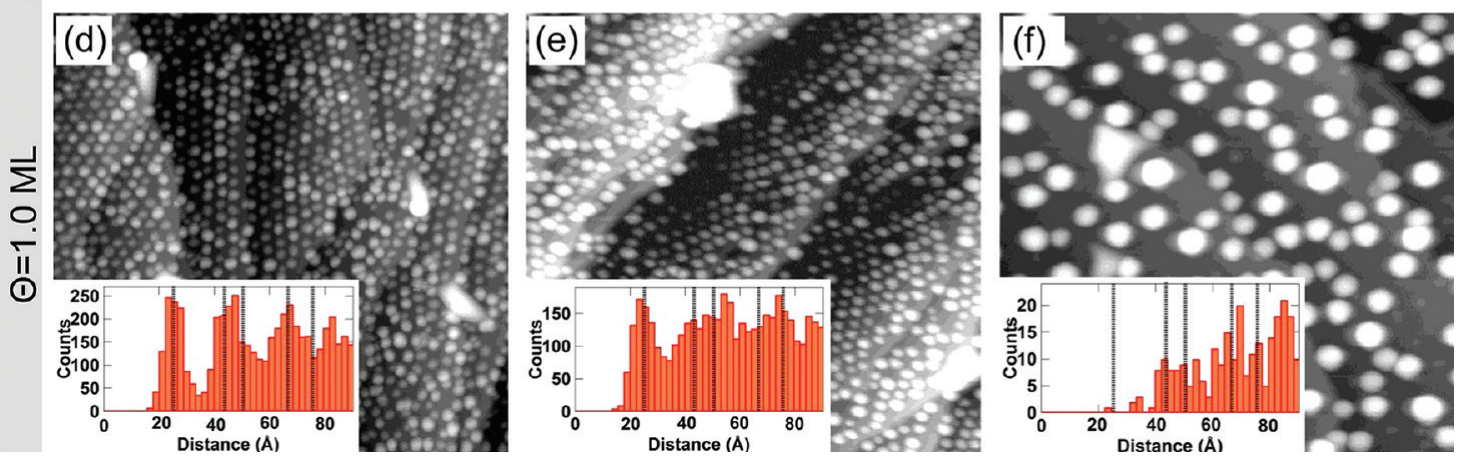

Figure 1. STM images $\left(800 \times 800 \AA^{2}\right)$ of Rh clusters on the graphene/lr(111). Radial distributions are displayed in the insets. Dotted vertical lines indicate the distances between $n$th nearest neighbor moirè cells. Scanning parameters: (a) $I=0.5 \mathrm{nA}, V_{B}=$ $+1.0 \mathrm{~V}$; (b) $I=0.5 \mathrm{nA}, V_{\mathrm{B}}=+0.5 \mathrm{~V}$; (c) $I=0.5 \mathrm{nA}, V_{\mathrm{B}}=+0.5 \mathrm{~V}$; (d) $I=0.15 \mathrm{nA}, V_{\mathrm{B}}=-0.2 \mathrm{~V}$; (e) $I=0.3 \mathrm{nA}, V_{\mathrm{B}}=+0.9 \mathrm{~V}$; (f) $I=0.5 \mathrm{nA}$, $V_{\mathrm{B}}=+0.5 \mathrm{~V}$.

has been first demonstrated in the case of Ir clusters on GR grown on $\operatorname{Ir}(111)^{12}$ and more recently on other substrates, ${ }^{13-19}$ and even in the case of molecules. ${ }^{20} \mathrm{~A}$ great advantage of using GR is that it is the strongest two-dimensional material known, a benchmark for structural and mechanical applications. ${ }^{21}$ Moreover, GR has exceptional heat transport properties ${ }^{22}$ and incomparably high electrical conductivity at room temperature ${ }^{23}$ originating from its unique electronic structure. This makes GR an ideal candidate for the production of nanocluster-based materials. ${ }^{24-26}$

The second exciting challenge to be faced, which we specifically address in the present work, is the understanding of the relationship between geometric and electronic structure of under-coordinated atoms in supported nanoclusters and how this varies with cluster size, shape, and density. At the nanometer scale, where the fractions of edge and facet atoms become no longer negligible, both surface energy and surface stress increase. This can result in large out-of-plane bond length contraction, as revealed in coherent X-ray diffraction experiments on Au nanocrystals, ${ }^{27}$ and thus modify the electronic and magnetic properties of the nanoclusters.

In order to shed light into these issues, we here combine high-energy resolution $\mathrm{X}$-ray photoelectron spectroscopy (XPS), scanning tunneling microscopy (STM), and density functional theory (DFT) to study the properties of under-coordinated atoms at graphene-supported Rh nanoclusters. Rhodium was selected among other elements because its nanoparticles exhibit high activity and excellent selectivity toward $\mathrm{NO}_{x}$ reduction and thus are used in car exhaust gas purification. Additionally, bulk $\mathrm{Rh}$ is very close to satisfying the Stoner criterion of ferromagnetism, ${ }^{28}$ and the increased density of states in low-dimensional structures is likely to stabilize the magnetic state in $\mathrm{Rh}$ nanoclusters. Recent experiments have indeed shown that 20 atom clusters embedded in inert xenon present total magnetic moments as large as $0.4 \mu_{\mathrm{B}}{ }^{29}$

\section{RESULTS AND DISCUSSION}

Rh clusters were grown on epitaxial GR on $\operatorname{Ir}(111)$. The metal surface was cleaned by using a well-established procedure which yields an ordered and clean surface, as proved by the sharp and low background $(1 \times 1)$ low energy electron diffraction (LEED) pattern and by the absence of traces of contaminants in the C 1s, S 2p, and O 1s core level regions.

Epitaxial GR was grown by means of chemical vapor deposition of ethylene. ${ }^{12,30}$ The quality of the GR layer was checked by the LEED pattern, which exhibits extra spots around the zero- and first-order diffraction spots of the substrate, a clear fingerprint of a moirè structure. LEED line profile analysis reveals that the moirè cell can be approximated as $(10 \times 10)$ GR unit cells over $(9 \times 9)$ Ir unit cells, with a periodicity of about $25 \AA$. Each moirè cell presents three different regions, whose 


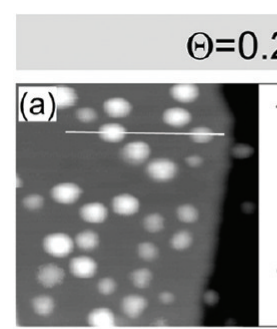

(b)
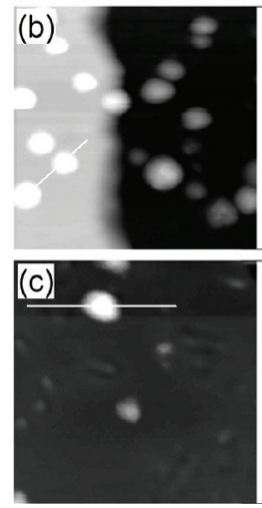
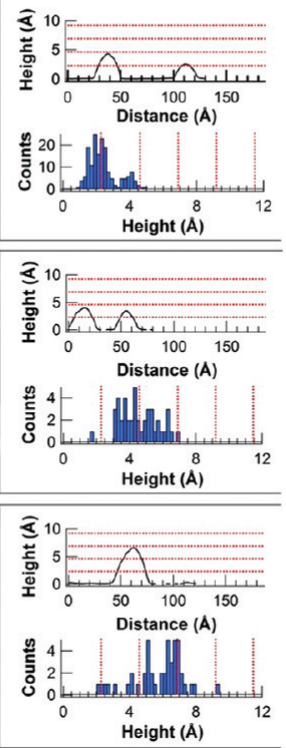

$\Theta=1.0 \mathrm{ML}$
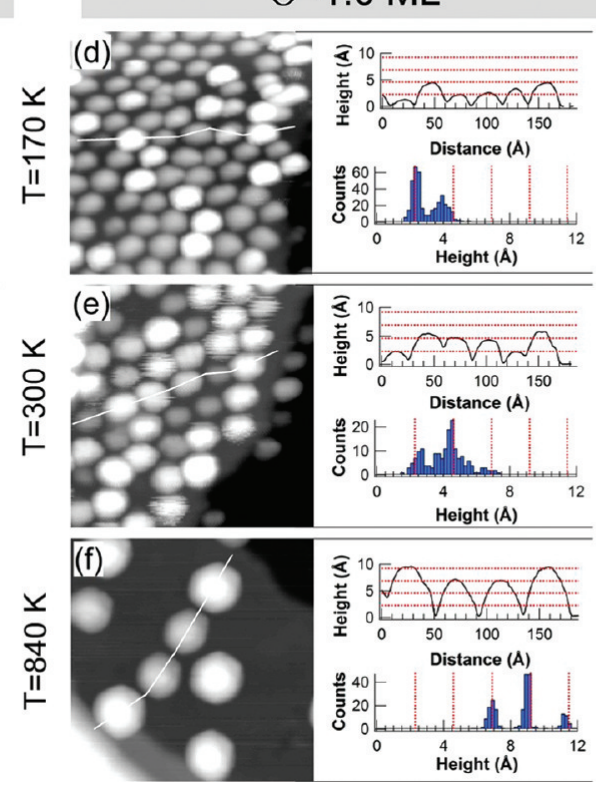

(g)
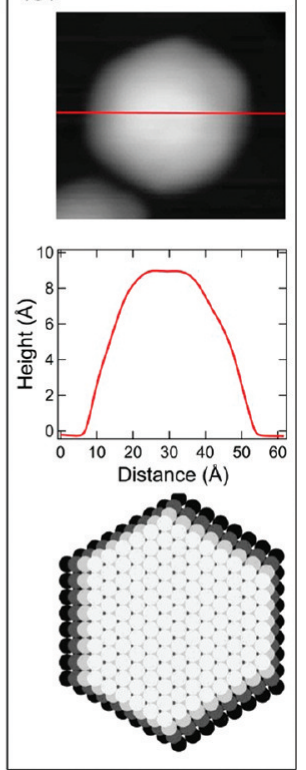

Figure 2. ( $a-f)$ Selected line profiles and cluster height histograms are displayed beside the STM images relative to the $200 \times 200 \AA^{2}$ STM images. Dotted red lines indicate the multiples of the (111) step height in bulk Rh geometry. (g) STM image $\left(6 \times 6 \mathrm{~nm}^{2}\right)$ of a single cluster obtained after annealing to $840 \mathrm{~K}$, its line profile and structural model. Scanning parameters: (a) $I=0.5 \mathrm{nA}, V_{\mathrm{B}}=+0.9 \mathrm{~V}$; (b) $I=0.2 \mathrm{nA}, V_{\mathrm{B}}=+0.3 \mathrm{~V}$; (c) $I=0.5 \mathrm{nA}, V_{\mathrm{B}}=+0.5 \mathrm{~V}$; (d) $I=0.5 \mathrm{nA}, V_{\mathrm{B}}=+0.5 \mathrm{~V}$; (e) $I=0.3 \mathrm{nA}, V_{\mathrm{B}}=+0.6 \mathrm{~V}$; (f) $I=0.5 \mathrm{nA}, V_{\mathrm{B}}=+0.5 \mathrm{~V}$; (g) $I=0.5 \mathrm{nA}, V_{\mathrm{B}}=+0.5 \mathrm{~V}$.

names-fcc, hcp, and atop-identify the nonequivalent adsorption sites of the C-rings. While in the atop region the Ir substrate atom lies right in the center of the carbon ring, the fcc region (hcp region) centers an Ir fcc site (hcp site) in the honeycomb lattice. ${ }^{12}$ It is important to underline that $\mathrm{GR} / \mathrm{Ir}(111)^{31,32}$ represents, in analogy with what observed on $\mathrm{Pt}(111){ }^{33}$ the hallmark of a low interacting and quasi-freestanding GR layer.

In order to characterize the equilibrium structure, the distribution, and the coalescence process of the $\mathrm{Rh}$ nanoclusters, we have initially performed STM measurements. Different amounts of Rh atoms were deposited at low temperature, $T=90 \mathrm{~K}$, and their evolution was monitored as a function of the annealing temperature in the range of $T=170-840 \mathrm{~K}$. In Figure 1, we present two series of measurements obtained after annealing to 170,300 , and $840 \mathrm{~K}$ for samples with different Rh coverages, $\Theta=0.20 \pm 0.04 \mathrm{ML}(\mathrm{a}-\mathrm{c})$ and $\Theta=1.0 \pm 0.2 \mathrm{ML}(\mathrm{d}-\mathrm{f})$.

Close inspection of the STM images shows that, for both low and high Rh coverages, the clusters grown after deposition and annealing to $170 \mathrm{~K}$ (Figure 1a,d) are not randomly distributed but present a regular and spatially ordered occupancy of the moirè cells. Each cell is occupied by a single cluster, which results in the formation of an extended superlattice. Previous investigations exclude the atop region as the preferential cluster adsorption site, ${ }^{34,35}$ but we cannot discriminate between hcp and fcc. Larger aggregates are sparse and located exclusively at substrate step edges.
Annealing to $300 \mathrm{~K}$ (Figure $1 \mathrm{~b}, \mathrm{e}$ ) increases the mobility especially of the smaller clusters, while it is not particularly effective for the larger ones, which maintain a moirè cell occupation probability close to 1 (Figure 1e). This is confirmed by the measured radial distribution, where the peak at about $25 \AA$ (the distance between nearest-neighboring preferential adsorption sites) decreases for the lower coverage case and not for the high coverage one. Therefore, the observed cluster density decrease is due to wholecluster diffusion/coalescence processes, as observed in the case of Ir clusters on the GR/Ir(111) interface, ${ }^{13}$ rather than to the energetically hindered atomic detachment from the clusters. As the cluster mobility is enhanced by further annealing, the superlattice periodicity gets lost: large clusters composed by a few hundred atoms appear in the high coverage case, and the average distance between clusters increases (see Figure 1f).

In order to determine how the structural modifications occur at different annealing temperatures for nonequivalent coverages, we analyzed the cluster heights. In Figure 2, we present the resulting height distributions together with representative line scans, which can be compared with the $\mathrm{Rh}(111)$ interlayer distance indicated by the dotted red lines. At low coverage and low annealing temperature (Figure 2a), the clusters heights correspond mainly to that of a single atomic layer. Upon annealing to $300 \mathrm{~K}$ (Figure $2 \mathrm{~b}$ ), the average cluster height distribution is broad and centered around two atomic layers: single 


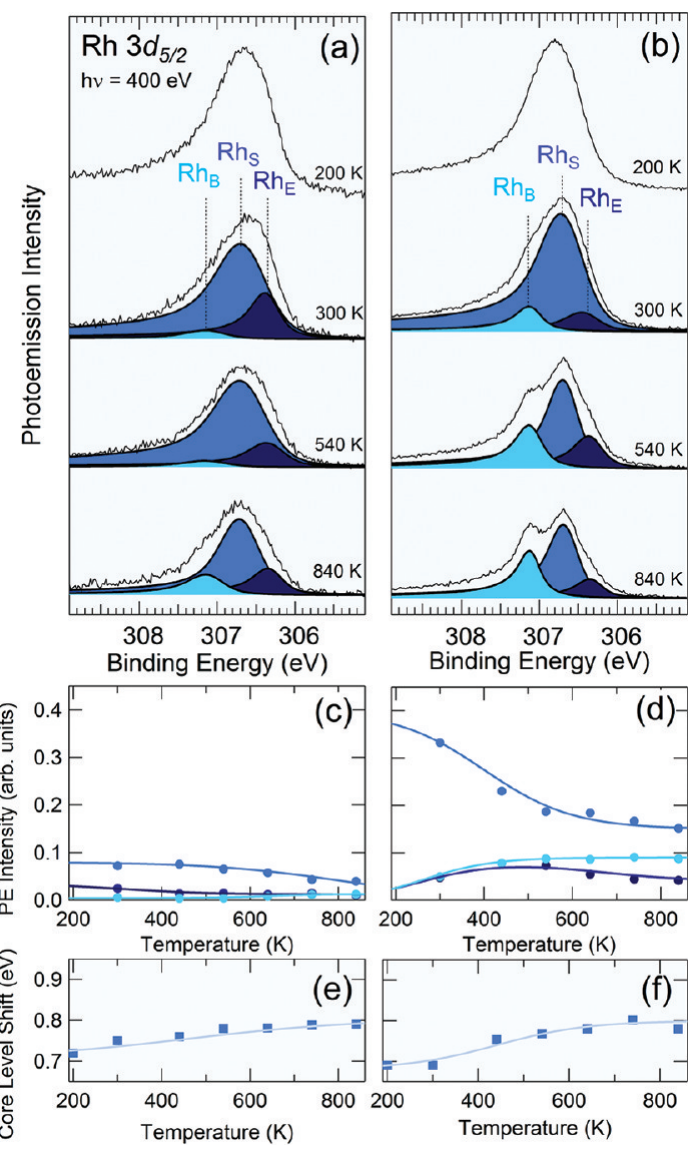

Figure 3. (Top panels) $\mathrm{Rh} 3 \mathrm{~d}_{5 / 2}$ core level spectra measured at normal emission ( $T=90 \mathrm{~K}$ ) corresponding to $\Theta=0.17$ (a) and $0.37 \mathrm{ML}(\mathrm{b})$, after annealing to the indicated temperatures. Dark blue, blue, and light blue curves indicate $\mathrm{Rh}$ atoms with different local coordination (see text for more details). (Middle panels) Temperature-dependent intensities of the $R h_{B}, R h_{S}$, and $R h_{E} R h^{3} d_{5 / 2}$ core level components. (Bottom panels) Temperature dependence of the binding energy of the $R h_{E}$ component expressed with respect to $R h_{B}$ fixed at $307.09 \mathrm{eV}$. Lines in (c), (d), (e), and (f) are only meant as guides for the eye.

layer clusters have almost completely vanished as a result of the diffusion/sintering process. The average height increases even more by annealing to $840 \mathrm{~K}$, a majority of clusters being three layers high (Figure 2c).

The morphological modifications of the clusters obtained with a larger initial coverage are reported in the central panels of Figure 2: in this case, the initial cell occupancy is clearly higher and the clusters are thicker on average after annealing to $300 \mathrm{~K}$. Interestingly, narrow peaks in the height distributions show that cluster heights are better defined than at low coverage and centered around integer numbers of (111) atomic layers.

This result suggests that each cluster presents a high degree of morphological order, which was not observed after annealing to lower temperature, $T=170 \mathrm{~K}$. Interestingly, after annealing to $840 \mathrm{~K}$, clusters clearly extend over more than one moirè unit cell and exhibit an ordered hexagonal shape (Figure $2 \mathrm{~g}$ ). While it is well-known that steps between large terraces can be revealed in STM, the same in general is not true for nanocluster interlayer terraces, ${ }^{36}$ which can be narrower that the tip radius (see Supporting Information). Despite the absence of atomic resolution on its flat top part, it is likely that the cluster has a truncated octahedron shape, thus forming lowest energy $\{111\}$ and $\{100\}$ nanofacets, as predicted by the Wulff theorem construction $^{37}$ and as supported by STM height distribution. A tentative model of the imaged cluster formed by $634 \mathrm{Rh}$ atoms arranged in 4 layers is reported in Figure $2 \mathrm{~g}$. As we will see later, this class of model clusters turns out to be in very good agreement with the results of the photoemission experiments (see Supporting Information).

To gain information on the electronic structure and the atomic distribution within Rh nanoclusters, we performed high-energy resolution and highly surface-sensitive XPS measurements complemented with theoretical calculations. Figure 3 shows $R h 3 d_{5 / 2}$ spectra, measured at selected annealing temperatures for different Rh coverages: (left panels) $\Theta=0.17 \pm 0.03 \mathrm{ML}$ and (right panels) $\Theta=0.37 \pm 0.06 \mathrm{ML}$.

All of the spectra obtained after annealing to temperatures higher than $200 \mathrm{~K}$ show three distinct components, $R h_{B}, R h_{S}$, and $R h_{E}$, with binding energies (BEs) of $307.09,306.67$, and $306.36 \mathrm{eV}$, respectively. The first of these peaks corresponds to the bulk component measured from Rh single crystals, ${ }^{38,39}$ and we therefore assign it to bulk-like atoms with high coordination number, $C_{N}=12$.

The core level shift (CLS) of $-485 \mathrm{meV}$ measured for the surface peak on $\mathrm{Rh}(111)^{39}$ supports the attribution of the $\mathrm{Rh}_{\mathrm{s}}$ component, shifted by $-420 \mathrm{meV}$, to $\mathrm{Rh}$ atoms placed at the cluster surface facets, with $C_{N}$ close to 9 . Finally, the comparison with the results of single adatoms and dimers deposited on $\{111\}$ and $\{100\} \mathrm{Rh}$ surfaces ${ }^{40}$ suggests that the third component, $\mathrm{Rh}_{\mathrm{E}}$, should be assigned to under-coordinated Rh atoms at the edges of the clusters, as will be confirmed later by DFT calculations. Oxygen contamination, both in terms of chemisorbed atoms or oxidic species, has the effect of increasing the $\mathrm{Rh} 3 \mathrm{~d}_{5 / 2}$ BE on Rh atoms. ${ }^{41,42}$ Other contamination sources have been thoroughly excluded via careful quantitative XPS.

By increasing the annealing temperature, we observe a progressive decrease of the overall photoemission signal, as evidenced in Figure $3 c, d$. This behavior, due to inelastic mean free path effects in 3D structures, is in agreement with the STM-observed cluster morphological evolution since we can unambiguously exclude both Rh evaporation and intercalation. For low coverage deposition, the intensity of the $\mathrm{Rh}_{B}$ component remains very low and grows appreciably only after annealing to a temperature higher than $600 \mathrm{~K}$. This suggests that below $600 \mathrm{~K}$ most of the 
clusters present two layers at maximum. Only at higher annealing temperatures does the $\mathrm{Rh}_{B}$ component increase, indicating the formation of $3 \mathrm{D}$ clusters with more than two layers, with Rh bulk-like coordinated atoms $\left(C_{N} \approx 12\right)$, in good agreement with the STM observation. A similar trend is observed for the high coverage case where, because of the higher Rh concentration, a low density of 3D clusters is found already after annealing to $T=300 \mathrm{~K}$.

What appears most remarkable is the high degree of crystallinity that clusters present when annealed to the highest temperature. While for the low coverage case all of the spectral components are characterized by quite a large Gaussian contribution, for the high coverage case, the three components become much narrower and a minimum appears between the $\mathrm{Rh}_{\mathrm{B}}$
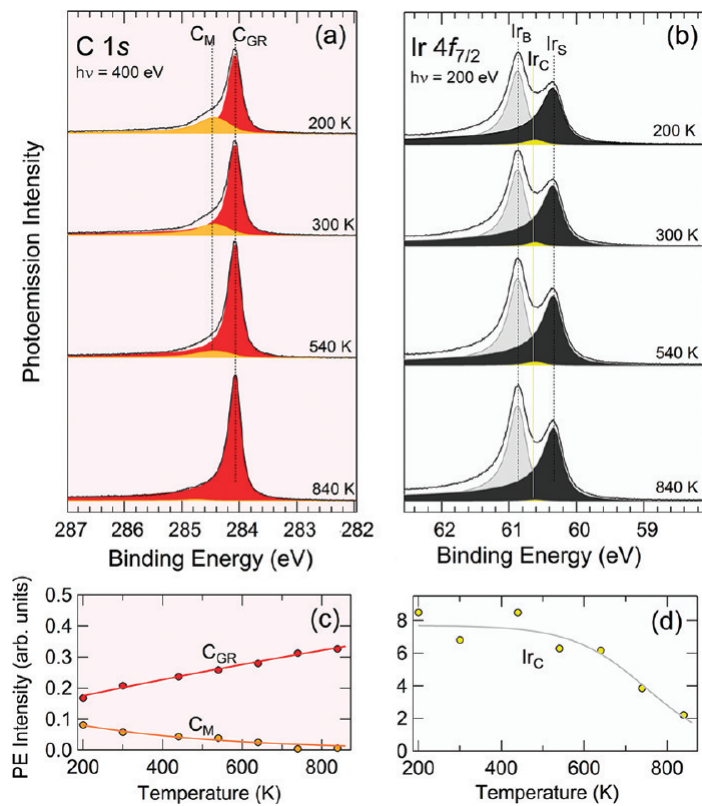

Figure 4. Thermal evolution of the (a) $C 1 s$ and (b) Ir $4 f_{7 / 2}$ core level spectra for Rh coverage equal to $0.37 \mathrm{ML}$. Thermal evolution of the (c) $C_{G R}$ and $C_{M}$ and (d) $\operatorname{Ir}_{C}$ components. Lines in (c) and (d) are only meant as guides for the eye. and $\mathrm{Rh}_{\mathrm{S}}$ photoemission peaks. The overall line shape strongly resembles that obtained for Rh single crystals. ${ }^{39}$ This suggests a change from clusters formed by a large variety of locally nonequivalent Rh atoms, resulting in a Gaussian inhomogeneous spectral broadening, to a configuration where each cluster has a high intrinsic structural order: monodispersed particles expose well-defined nanofacets mainly oriented along the $\{111\}$ crystallographic plane. Again, this is in very good agreement with the STM results on the cluster morphology and cluster height distribution. Noteworthy is the behavior of the binding energy of the $\mathrm{Rh}_{\mathrm{E}}$ component with respect to the $\mathrm{Rh}_{\mathrm{B}}$ contribution: for both coverages, the shift increases with increasing annealing temperature as we show in Figure 3e,f.

Figure 4 shows selected $C 1 \mathrm{~s}$ and $\operatorname{Ir} 4 \mathrm{f}_{7 / 2}$ core level spectra corresponding to the high Rh coverage described in Figure 3b. At low annealing temperature, the C 1s spectrum presents a narrow component at $284.06 \mathrm{eV}$ $\left(\mathrm{C}_{\mathrm{GR}}\right.$, red curve), originated by the $\mathrm{sp}^{2}$ carbon atom configurations of the unperturbed $\mathrm{GR}^{31,32}$ and $\mathrm{a}$ shoulder at $284.37 \mathrm{eV}\left(\mathrm{C}_{\mathrm{M}}\right.$, orange curve), a sign of metal-GR bond formation. With increasing annealing temperature, the lower $\mathrm{BE}$ component increases in intensity at the expense of the higher BE peak, which disappears after annealing to $T=840 \mathrm{~K}$. The $\mathrm{Ir}_{4 \mathrm{f}} \mathrm{f/2}$ core level spectrum presents a similar trend: the intensity of the $\mathrm{Ir}_{\mathrm{S}}$ surface component at $60.31 \mathrm{eV}$ due to first-layer Ir atoms ${ }^{32,43}$ (black curve) increases with increasing temperature, while the small component $\operatorname{Ir}_{C}$ (yellow curve), lying between the bulk $\operatorname{Ir}_{\mathrm{B}}$ (gray curve) and surface $1 \mathrm{r}_{\mathrm{S}}$ peaks, disappears after annealing to $840 \mathrm{~K}$.

Two effects may contribute to the observed spectral evolution. The first one is the coalescence of Rh atomic aggregates into larger clusters, which reduces the carbon-covered area and increases the Ir surface component. The second one is the reduced interaction of the larger cluster with the carbon network underneath: the metal cohesive energy contribution exceeds the

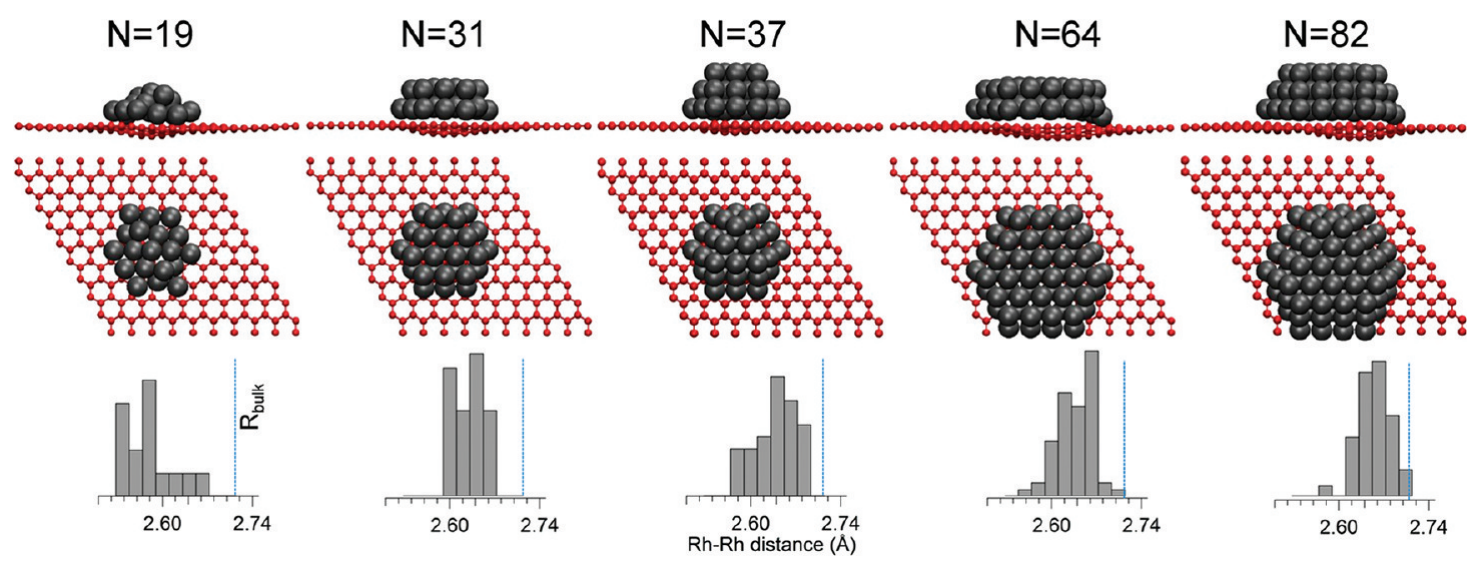

Figure 5. Top and side views of the calculated structural models for Rh clusters supported by GR formed by $N=19,31,37,64$, and $82 \mathrm{Rh}$ atoms. The evolution of the $\mathrm{Rh}-\mathrm{Rh}$ bond length distribution with cluster size morphology is reported in the histograms below (the vertical line at $2.715 \AA$ A marks the $\mathrm{Rh}-\mathrm{Rh}$ bulk distance). 


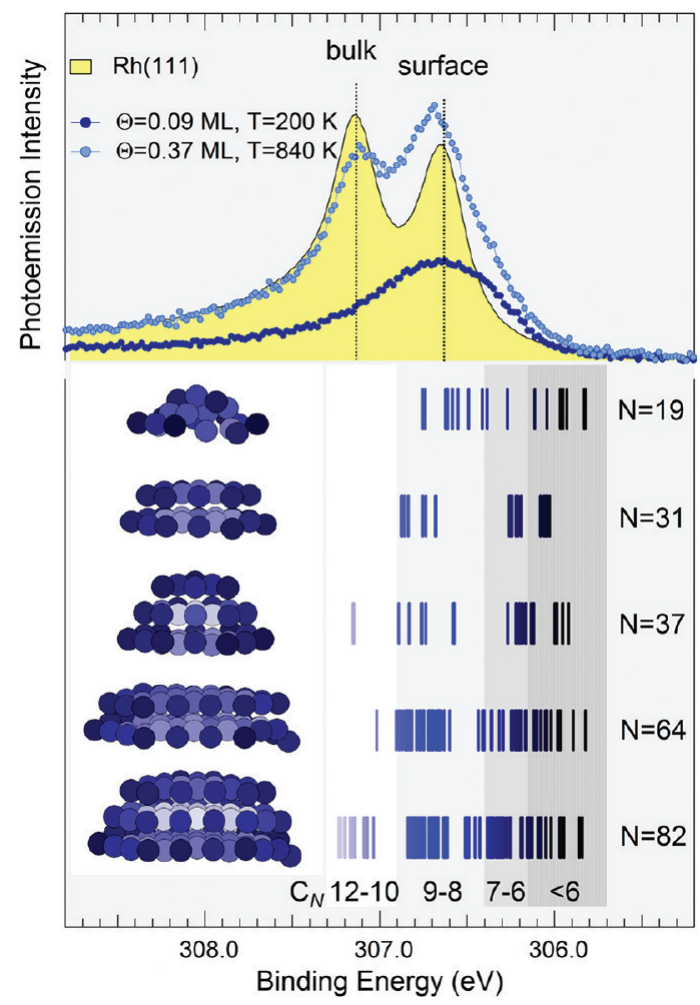

Figure 6. $R h 3 d_{5 / 2}$ spectra of $R h(111), 0.09 \mathrm{ML} R h$ on GR/ $\operatorname{Ir}(111)$ annealed at $200 \mathrm{~K}, 0.37 \mathrm{ML}$ Rh on GR/Ir(111) annealed at $840 \mathrm{~K}$. The barcodes in the bottom panel represent the calculated $\mathrm{Rh} 3 \mathrm{~d}_{5 / 2}$ BEs for all of the 233 nonequivalent atomic configurations of the simulated $\mathrm{Rh}$ nanoclusters.

metal-carbon interaction with increasing average number of Rh atoms per cluster. The latter effect is compatible with the results of theoretical calculations, which show that small Ir clusters grown on GR/Ir(111) pin the GR layer to the underlying metal, as a result of a local $\mathrm{sp}^{3}$-bond formation. ${ }^{34}$ In line with these findings, our data suggest that $\mathrm{sp}^{3}$ rehybridization is reduced with an increase of the Rh cluster size.

In order to reach a quantitative understanding of the relationship between the nanocluster geometric structure and their electronic properties, we have performed in-depth DFT calculations on clusters characterized by nonequivalent sizes and morphologies. In particular, we studied clusters formed by $N$ Rh atoms, with $N=19,31,37,64$, and 82 , disposed on two and three layers. The structural models obtained after relaxation of the carved bulk geometry are shown in Figure 5 . For $N=19$, the smallest cluster examined, it is evident that a clear layered structure is missing. Even though a second layer is not yet completely formed, its geometry reflects the preference for higher coordination numbers. This finding is in good agreement with XPS analysis, which reports broad $\mathrm{Rh} 3 \mathrm{~d}_{5 / 2}$ peaks, and with STM height distribution measurements, which exhibit a peak most probably originated by small flat clusters and a second peak due to corrugated clusters less than two layers height. In this case, the calculated average $\mathrm{Rh}-\mathrm{Rh}$ atomic bond length is shorter than 2.6 ̊. Two- and three-layer clusters formed by a larger number of atoms are energetically stable and are associated with the presence of flat top nanofacets oriented along the $\{111\}$ plane. The weight of $\mathrm{Rh}-\mathrm{Rh}$ bond length distribution (Figure 5, bottom) moves toward larger values. Besides the flat top hexagonal facet, the largest simulated cluster presents also $\{100\}$ and $\{111\}$ tilted nanofacets. For this cluster, the average $\mathrm{Rh}-\mathrm{Rh}$ distance is equal to $2.66 \AA$, still lower than the interatomic Rh-Rh bulk distance of $2.715 \AA$.

For an unambiguous assignment of the experimental Rh $3 d_{5 / 2}$ components and for a thorough description of the nanocluster electronic properties, we have performed theoretical calculations of the CLSs for a total of 233 nonequivalent atomic configurations of the simulated Rh nanoclusters. The results are shown in Figure 6 as barcodes, together with two of the measured $\mathrm{Rh} 3 \mathrm{~d}_{5 / 2}$ spectra corresponding to $\Theta=0.09 \mathrm{ML}$ after annealing to $T=200 \mathrm{~K}$ and $\Theta=0.37 \mathrm{ML}$ after annealing to $T=840 \mathrm{~K}$, as dark and light blue lines, respectively. The spectrum corresponding to a $\mathrm{Rh}(111)$ single crystal is also displayed for comparison. Structural models are also reported with the color scale reflecting the $B E s$ of the $R h 3 d_{5 / 2}$ core levels.

The results clearly show that $\mathrm{Rh}$ atoms with high coordination number $C_{N}$ are associated with high $\mathrm{BE}$ values: only three-layer clusters present $B E s$ larger than $307 \mathrm{eV}$. At the other extreme, BEs smaller than $306.4 \mathrm{eV}$ are fingerprints of edge atoms with 7 or less nearest neighbors. These atoms are not present at the surface of $\mathrm{Rh}(111)$, and therefore their contribution does not appear in the corresponding single crystal spectrum. In the intermediate $B E$ range (306.4-307.0 eV), we find mainly atoms of top and bottom $\{111\}$ nanofacets presenting $C_{N}=9$. Indeed, the light blue spectrum of Figure 6 presents a peak in this energy range.

To highlight the link between the local geometric configuration and the corresponding electronic structure, we have plotted the core level shift data for all of the different atomic configurations as a function of their effective coordination number $C_{\mathrm{E}}{ }^{40}$ which takes into account both the effects of the coordination number $C_{N}$ and of the local bond strain, that is, the changes of the $\mathrm{Rh}-\mathrm{Rh}$ average distance. This definition naturally assigns larger contributions to closer atoms and smoothly phases out farther ones. $C_{\mathrm{E}}(i)$ is defined as $\sum_{\mathrm{j}} \rho_{\mathrm{Rh}}^{\mathrm{at}}\left(R_{i j}\right) / \rho_{\mathrm{Rh}}^{\text {at }}\left(R_{\text {bulk }}\right)$, where the sum is calculated over all of the nearest neighbors $j$ of atom $i$ and $\rho_{\mathrm{Rh}}^{\text {at }}$ is the calculated spherical charge density distribution of an isolated Rh atom as a function of the distance $R$ from the nucleus. $R_{i j}$ is the distance of nearest neighbor $j$ from atom $i$, and $R_{\text {bulk }}$ is the bulk interatomic distance. Figure 7a shows that $C_{E}$ is consistently larger than $C_{N}$ (red line), and nanoclusters therefore present significantly reduced bond lengths. The data reported in Figure $7 \mathrm{~b}$ show a linear behavior of the CLS versus $C_{\mathrm{E}}$, 


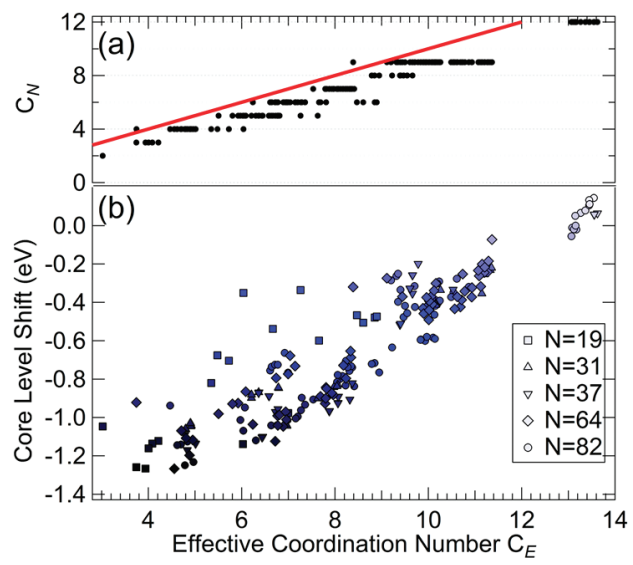

Figure 7. (a) Nominal coordination number $C_{N}$ plotted against calculated effective coordination numbers $C_{E}$ for clusters with $N=19,31,37,64,82$. The red curve correspond to an ideal $C_{N}=C_{E}$. (b) Calculated CLSs as a function of the calculated effective coordination number $C_{E}$ for all the 233 nonequivalent atomic configurations of the simulated Rh nanoclusters.

except for a small number of scattered data points, most of which originate from $\mathrm{Rh}$ atoms sitting at the interface between the clusters and the GR layer, whose $B E$ is slightly influenced by the interaction with the $C$ atoms. Actually, the linear dependence suggests that core levels in nanoclusters are very sensitive to both coordination, as predicted by a simple tight-binding model, and bond length contraction. The CLS changes upon annealing temperature, reported in Figure 3e,f, are in line with these findings and can be explained as due to variations of the average interatomic distance for edge atoms with the cluster size. This suggests that structural relaxation may take place also in the case of a very small GR-supported Rh cluster. Very interestingly, a similar contraction of bond lengths of outer atoms has been measured in $\mathrm{Au}$ nanocrystals with sizes between 3 and $5 \mathrm{~nm}^{27}$ While the contraction of interatomic distances of first-layer atoms is a phenomenon typically observed on solid surfaces, which has deep effects on surfaces properties like surface thermal stability and chemical reactivity, its detection on supported nanoclusters is extremely difficult.

The link between nanocluster morphology, local bond length, electronic structure, and chemical reactivity is further deduced by plotting the $\mathrm{Rh} 3 \mathrm{~d}_{5 / 2} \mathrm{CLSs}$ versus the calculated Rh d-band center $\varepsilon_{\mathrm{d}}$. The latter is an extremely important parameter that affects many different nanoscale properties, including chemical reactivity and magnetism. For example, in the HammerNørskov ${ }^{44,45}$ model of chemical reactivity, the center of the $d$-band characterizes the ability of the surface d-electrons to participate in the bond formation with adsorbates. Since the d-band center depends on the surroundings of a given atom, one possibility for modifying the reactivity of a metal is by depositing

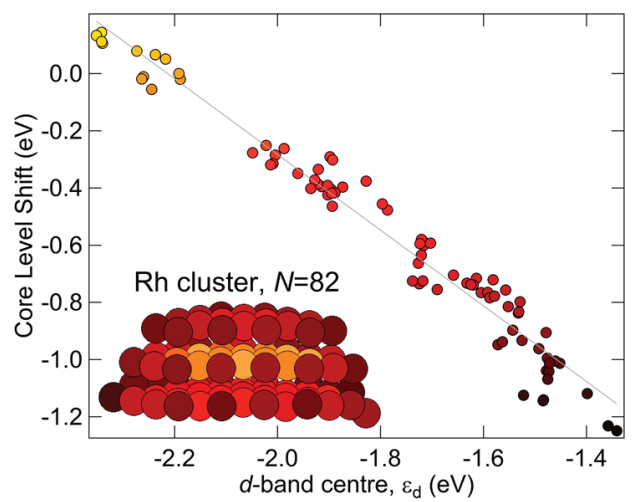

Figure 8. Calculated CLSs as a function of the calculated d-band center for all of the atomic configurations of the Rh nanocluster formed by 82 atoms.

overlayers, making alloys or, as in the present case, by tuning the interatomic distances at surfaces.

The remarkable linear relationship between core level shifts and $\varepsilon_{\mathrm{d}}$, obtained for the largest cluster reported in Figure 8, confirms the possibility to probe electronic structural properties in nanoclusters via XPS. The core level spectral distribution moves toward lower BEs as we increase the fraction of atoms sitting at facets, edges, and kinks and therefore under-coordinated. However, this trend is counteracted upon by the contraction of bond lengths because of the small dimension of the cluster, which pushes the BEs toward higher values. Therefore, these two competing contributions result in the critically important determination and tuning of nanocluster chemical reactivity.

Finally, to inspect the magnetic properties of the clusters, we performed spin-polarized DFT calculations on the 31 and 82 atom clusters, keeping the geometries fixed to those obtained with the nonmagnetic calculations. We find that the 82 atom cluster displays a total magnetic moment of only $2.62 \mu_{\mathrm{B}}\left(0.03 \mu_{\mathrm{B}}\right.$ /atom), while the 31 atom cluster has a significantly higher moment of $5.36 \mu_{\mathrm{B}}\left(0.17 \mu_{\mathrm{B}}\right.$ /atom). This suggests that a narrow size distribution of clusters grown on GR could provide the first observation of a non-zero total magnetic moment for surface-supported Rh clusters, with important consequences with respect to the magnetic anisotropy and the definition of a preferential axis of magnetization. In fact, previous findings of a magnetic signal were obtained on free Rh clusters ${ }^{46}$ and on Rh clusters embedded in inert xenon, ${ }^{29}$ that is, in both cases for clusters in isotropic environments.

\section{CONCLUSIONS}

We reported on a combination of STM imaging, high-energy resolution core level spectroscopy, and density functional theory, providing new and valuable information about the relationship between structural and electronic properties of nanoclusters supported by epitaxial GR. The finite size effects associated with the miniaturization of metal clusters can result in 
enhanced chemical activities and magnetic moments. By carefully selecting the Rh coverage and the annealing temperature, we have demonstrated the possibility to control the morphology of Rh nanoclusters. This determines the density of edge and facet atoms and the nanocluster arrangement in superlattices. In particular, growth conditions of the nanoclusters exhibit a remarkably high degree of crystallinity. We suggest that our small nanoclusters could also be used as seeds for the formation of larger Rh nanoaggregates (diameter between 10 and $100 \mathrm{~nm}$ ) with a spherical shape, which have been observed to spontaneously form hierarchical assemblies with high thermal stability. ${ }^{47}$

The preparation route we have employed could be extended to other metals, thus offering a new way to design and grow GR-based materials in a precise and reproducible way. Our approach gives access to detailed information on fundamental properties at the nanoscale and may help pave the road for attractive technological applications in the field of catalysis and magnetism.

\section{METHODS}

Experimental Methods. Rh atoms were deposited at low temperature on epitaxial graphene using a high-purity filament that was heated and then kept for a specific time at a temperature corresponding to a deposition rate of 0.03 monolayers (ML)/ min. The pressure in the UHV systems was always below $4 \times 10^{-10}$ mbar during deposition, thus preventing the adsorption of impurities on the surface.

The photoemission experiments were performed at the SuperESCA beamline of the Elettra synchrotron radiation source. $\mathrm{Rh} 3 \mathrm{~d}_{5 / 2}$ and $C 1 \mathrm{~s}$ core level measurements were performed at normal emission using $400 \mathrm{eV}$ photon energy and with an overall experimental resolution of $50 \mathrm{meV}$. Ir $4 \mathrm{f}_{7 / 2}$ spectra were measured at $200 \mathrm{eV}$. All of the BEs were calibrated with respect to the Fermi level, measured under the same conditions. A Phoibos hemispherical electron energy analyzer was used to collect the spectra. During the measurements, the sample was kept at $30 \mathrm{~K}$ to reduce phonon-induced broadening of the spectral lines. Rh coverages have been estimated from C $1 \mathrm{~s}$ and $R h 3 d_{5 / 2}$ spectral intensities normalized by their respective cross sections and accounting for the limited inelastic mean free path. The analysis of the spectra was performed using a Doniach-Sùnjić function characterized by a Lorentzian width $\Gamma$, which takes into account the effect of finite core hole lifetime, and by the asymmetry index $\alpha$, which describes the low-energy electron hole pair excitations at the Fermi level, convoluted with a Gaussian distribution (full width at halfmaximum $G$ ), to take into account phonon, inhomogeneous, and instrumental broadening. The background was always assumed to be linear.

The STM experiments were performed in a UHV chamber, equipped with standard sample preparation facilities and with a variable-temperature Omicron VT-STM. Measurements were carried out at $T<173 \mathrm{~K}$, with a tunneling current in the $0.1-1.0 \mathrm{nA}$ range and the sample biased by +0.2 to $+0.9 \mathrm{~V}$ to probe its empty states. The GR substrate was prepared as described above, and $\mathrm{Rh}$ atoms were deposited while keeping the sample at $90 \mathrm{~K}$. The amount of Rh deposited has been estimated considering the multilayer growth of the clusters and is expressed in units of the complete atomic Rh layer. The uncertainties are mainly due to the imperfect knowledge of the cluster and tip apex shape.

Theoretical Methods. DFT calculations were performed with the VASP code $^{48}$ using the Perdew-Burke-Ernzerhof exchange-correlation functional, ${ }^{49}$ and adopting an efficient extrapolation method for the charge density. ${ }^{50}$ The interactions between electrons and ions were described using the projectoraugmented wave method. ${ }^{51,52}$ For Rh and C, we used the $5 s^{1} 4 d^{8}$ and the $2 s^{2} 2 p^{2}$ valence electronic configurations, respectively. Single particle orbitals were expanded in plane waves, with a cutoff energy of $400 \mathrm{eV}$. We adsorbed the Rh clusters on an isolated sheet of GR, in a $(10 \times 10)(200$ carbon atoms $)$ periodic surface unit cell. The clusters were initially prepared by carving the perfect crystal. Equilibrium structures were then obtained by relaxing all of the atoms in the unit cell until the residual forces were less than $0.02 \mathrm{eV} / \AA$, using the $\Gamma$ point only. $R \mathrm{~h} 3 \mathrm{~d}_{5 / 2}$ core level BEs were calculated including final state effects and using a $(4 \times 4 \times 1)$ grid of $\mathbf{k}$-points.

The partial density of states $n_{l m}^{i}(E)$ of atom $i$ is defined as the projection of the density of states onto spherical harmonics $Y_{I m}^{i}$ centered on the atom: $n_{I m}^{i}(E)=\Sigma_{N} \int_{B Z} \mathrm{~d} \mathbf{k}\left|\left\langle Y_{I m}^{i} \mid \psi_{N \mathbf{k}}\right\rangle\right|^{2}$ $\delta\left(E-E_{N \mathbf{k}}\right)$, with the projection $\left\langle Y_{I m}^{i} \mid \psi_{N \mathbf{k}}\right\rangle$ calculated by integrating over a sphere of radius $R_{i}=1.4 \AA$ (for Rh) centered on atom $i$. Here $\psi_{N k}$ is the crystal wave function of band $N$ at wave-vector $k$ and $E_{N k}$ is the corresponding energy eigenvalue. We define the $p$ th moment of the density of states $n_{l m}^{i}(E)$ as $\mu_{p}=\int \mathrm{d} E E^{p} n_{l m}^{i}(E)$; $\mu_{0}$ and $\mu_{1} / \mu_{0}$ give the total number of states in the band and the band center $B_{l m}^{i}$, respectively. In particular, we define the d-band center $B_{\mathrm{d}}^{i}$ of atom $i$ as the ratio $\mu_{1} / \mu_{0}$ obtained from the partial density of states $n_{\mathrm{d}}^{i}(E)=\sum_{m=-2}^{2} n_{2 m}^{i}(E)$.

Conflict of Interest: The authors declare no competing financial interest.

Acknowledgment. The work of D.A. and M.P. was conducted as part of a EURYI scheme award as provided by EPSRC (see www.esf.org/euryi). Calculations were performed on the HECToR national service (UK) and on JaguarPF at the National Center for Computational Science (USA). This research used resources of the Oak Ridge Leadership Computing Facility located in the Oak Ridge National Laboratory, which is supported by the Office of Science of the Department of Energy under Contract DE-AC0500OR22725. D.A. and A.B. acknowledge the Royal Society for support. A.B. acknowledges the Università degli Studi di Trieste for the Finanziamento per Ricercatori di Ateneo. A.C. is grateful to $\mathrm{H}$. Brune for supporting the present project. A.C. acknowledges the Collegio Universitario per le Scienze L. Fonda for support. C.A. and C.D. acknowledge support from CNR through the ESF FANAS project NOMCIS. C.A. acknowledges ECP FP7 under Grant Agreement No. 212348 NFFA.

Supporting Information Available: We support the case for the cluster model presented in Figure $2 \mathrm{~g}$ and present a complementary statistical analysis of nanocluster morphological properties obtained by comparison of STM and XPS information. This material is available free of charge via the Internet at http://pubs.acs.org.

\section{REFERENCES AND NOTES}

1. Meiwes-Broer, K.-H., Ed. Metal Clusters at Surfaces, Structure, Quantum Properties, Physical Chemistry; Springer: Berlin, 2000.

2. Li, Y.; Somorjai, G. A. Nanoscale Advances in Catalysis and Energy Applications. Nano Lett. 2010, 10, 2289-2295.

3. Haruta, M.; Yamada, N.; Kobayashi, T.; lijima, S. Gold Catalysts Prepared by Coprecipitation for Low-Temperature Oxidation of Hydrogen and of Carbon Monoxide. J. Catal. 1989, 115, 301-309.

4. Valden, M.; Lai, X.; Goodman, D. W. Onset of Catalytic Activity of Gold Clusters on Titania with the Appearance of Nonmetallic Properties. Science 1998, 281, 1647-1650. 
5. Campbell, C. T. The Active Site in Nanoparticle Gold Catalysis. Science 2004, 306, 234-235.

6. Hvolbæk, B.; Janssens, T. V.; Clausen, B. S.; Falsig, H.; Christensen, C. H.; Nørskov, J. K. Catalytic Activity of Au Nanoparticles. Nano Today 2007, 2, 14-18.

7. Tian, N.; Zhou, Z.-Y.; Sun, S.-G.; Ding, Y.; Wang, Z. L. Synthesis of Tetrahexahedral Platinum Nanocrystals with HighIndex Facets and High Electro-oxidation Activity. Science 2007, 316, 732-735.

8. Vajda, S.; Pellin, M. J.; Greeley, J. P.; Marshall, C. L.; Curtiss, L. A.; Ballentine, G. A.; Elam, J. W.; Catillon-Mucherie, S.; Redfern, P. C.; Mehmood, F.; et al. Subnanometre Platinum Clusters as Highly Active and Selective Catalysts for the Oxidative Dehydrogenation of Propane. Nat. Mater. 2009, 8, 213-216.

9. Gambardella, P.; Rusponi, S.; Veronese, M.; Dhesi, S. S.; Grazioli, C.; Dallmeyer, A.; Cabria, I.; Zeller, R.; Dederichs, P. H.; Kern, K.; et al. Giant Magnetic Anisotropy of Single Cobalt Atoms and Nanoparticles. Science 2003, 300, 11301133.

10. Rusponi, S.; Cren, T.; Weiss, N.; Epple, M.; Buluschek, P.; Claude, L.; Brune, $\mathrm{H}$. The Remarkable Difference between Surface and Step Atoms in the Magnetic Anisotropy of Two-Dimensional Nanostructures. Nat. Mater. 2003, 2, 546-551.

11. Wen, T.; Majetich, S. A. Ultra-Large-Area Self-Assembled Monolayers of Nanoparticles. ACS Nano 2011, 5, 88688876.

12. N'Diaye, A. T.; Bleikamp, S.; Feibelman, P. J.; Michely, T. Two-Dimensional Ir Cluster Lattice on a Graphene Moiré on $\operatorname{Ir}(111)$. Phys. Rev. Lett. 2006, 97, 215501.

13. N'Diaye, A. T.; Gerber, T.; Busse, C.; Mysliveček, J.; Coraux, J.; Michely, T. A Versatile Fabrication Method for Cluster Superlattices. New J. Phys. 2009, 11, 103045.

14. Zhou, Z.; Gao, F.; Goodman, D. W. Deposition of Metal Clusters on Single-Layer Graphene/Ru(0001): Factors That Govern Cluster Growth. Surf. Sci. 2010, 604, L31-L38.

15. Cui, Y.; Fu, Q.; Zhang, H.; Bao, X. Formation of Identical-Size Graphene Nanoclusters on Ru(0001). Chem. Commun. 2011, 47, 1470-1472.

16. Liao, Q.; Zhang, H. J.; Wu, K.; Li, H. Y.; Bao, S. N.; He, P. Nucleation and Growth of Monodispersed Cobalt Nanoclusters on Graphene Moiré on Ru(0001). Nanotechnology 2011, 22, 125303.

17. Pan, Y.; Gao, M.; Huang, L.; Liu, F.; Gao, H.-J. Directed SelfAssembly of Monodispersed Platinum Nanoclusters on Graphene Moirè Template. Appl. Phys. Lett. 2009, 95, 093106.

18. Sicot, M.; Bouvron, S.; Zander, O.; Rüdiger, U.; Dedkov, Y. S.; Fonin, M. Nucleation and Growth of Nickel Nanoclusters on Graphene Moirè on Rh(111). Appl. Phys. Lett. 2010, 96, 093115.

19. Murata, Y.; Petrova, V.; Kappes, B. B.; Ebnonnasir, A.; Petrov, I.; Xie, Y.-H.; Ciobanu, C. V.; Kodambaka, S. Moirè Superstructures of Graphene on Faceted Nickel Islands. ACS Nano 2010, 4, 6509-6514.

20. Mao, J.; Zhang, H.; Jiang, Y.; Pan, Y.; Gao, M.; Xiao, W.; Gao, H.-J. Tunability of Supramolecular Kagome Lattices of Magnetic Phthalocyanines Using Graphene-Based Moiré Patterns as Templates. J. Am. Chem. Soc. 2009, 131, 1413614137.

21. Lee, C.; Wei, X.; Kysar, J. W.; Hone, J. Measurement of the Elastic Properties and Intrinsic Strength of Monolayer Graphene. Science 2008, 321, 385-388.

22. Seol, J. H.; Jo, I.; Moore, A. L.; Lindsay, L.; Aitken, Z. H.; Pettes, M. T.; Li, X.; Yao, Z.; Huang, R.; Broido, D.; et al. TwoDimensional Phonon Transport in Supported Graphene. Science 2010, 328, 213-216.

23. Geim, A. K. Graphene: Status and Prospects. Science 2009, 324, 1530-1534.

24. Guo, S.; Wen, D.; Zhai, Y.; Dong, S.; Wang, E. Platinum Nanoparticle Ensemble-on-Graphene Hybrid Nanosheet: One-Pot, Rapid Synthesis, and Used as New Electrode Material for Electrochemical Sensing. ACS Nano 2010, 4, 3959-3968.
25. Yoo, E.; Okata, T.; Akita, T.; Kohyama, M.; Nakamura, J.; Honma, I. Enhanced Electrocatalytic Activity of Pt Subnanoclusters on Graphene Nanosheet Surface. Nano Lett. 2009, 9, 2255.

26. Venkateswara Rao, C.; Cabrera, C. R.; Ishikawa, Y. GrapheneSupported Pt-Au Alloy Nanoparticles: A Highly Efficient Anode for Direct Formic Acid Fuel Cells. J. Phys. Chem. C 2011, 115, 21963-21970.

27. Huang, W. J.; Sun, R.; Tao, J.; Menard, L. D.; Nuzzo, R. G.; Zuo, J. M. Coordination-Dependent Surface Atomic Contraction in Nanocrystals Revealed by Coherent Diffraction. Nat. Mater. 2008, 7, 308-313.

28. Cho, J.-H.; Scheffler, M. Surface Relaxation and Ferromagnetism of Rh(001). Phys. Rev. Lett. 1997, 78, 1299-1302.

29. Sessi, V.; Kuhnke, K.; Zhang, J.; Honolka, J.; Kern, K.; Tieg, C.; Šipr, O.; Minár, J.; Ebert, H. Intrinsic Orbital and Spin Magnetism in Rh Clusters on Inert Xenon Matrices. Phys. Rev. B 2010, 82, 184413.

30. Lizzit, S.; Baraldi, A. High-Resolution Fast X-ray Photoelectron Spectroscopy Study of Ethylene Interaction with Ir(111): From Chemisorption to Dissociation and Graphene Formation. Catal. Today 2010, 154, 68-74.

31. Preobrajenski, A.; Ng, M.; Vinogradov, A.; Mårtensson, N. Controlling Graphene Corrugation on Lattice-Mismatched Substrates. Phys. Rev. B 2008, 78, 073401.

32. Lacovig, P.; Pozzo, M.; Alfè, D.; Vilmercati, P.; Baraldi, A.; Lizzit, S. Growth of Dome-Shaped Carbon Nanoislands on Ir(111): The Intermediate between Carbidic Clusters and Quasi-Free-Standing Graphene. Phys. Rev. Lett. 2009, 103, 166101.

33. Merino, P.; Švec, M.; Pinardi, A. L.; Otero, G.; Martìn-Gago, J. A. Strain-Driven Moirè Superstructures of Epitaxial Graphene on Transition Metal Surfaces. ACS Nano 2011, 5, 5627-5634.

34. Feibelman, P. J. Pinning of Graphene to $\operatorname{Ir}(111)$ by Flat Ir Dots. Phys. Rev. B 2008, 77, 165419.

35. N'Diaye, A. T.; Coraux, J.; Plasa, T. N.; Busse, C.; Michely, T. Structure of Epitaxial Graphene on $\operatorname{Ir}(111)$. New J. Phys. 2008, 10, 043033.

36. Henry, C. R. Morphology of Supported Nanoparticles. Prog. Surf. Sci. 2005, 80, 92-116.

37. Wulff, G. Z. Kristallogr. 1901, 449-530.

38. Andersen, J. N.; Hennig, D.; Lundgren, E.; Methfessel, M.; Nyholm, R.; Scheffler, M. Surface Core-Level Shifts of Some 4d-Metal Single-Crystal Surfaces: Experiments and $A b$ Initio Calculations. Phys. Rev. B 1994, 50, 17525-17533.

39. Baraldi, A. Structure and Chemical Reactivity of Transition Metal Surfaces as Probed by Synchrotron Radiation Core Level Photoelectron Spectroscopy. J. Phys.: Condens. Matter 2008, 20, 093001.

40. Baraldi, A.; Bianchettin, L.; Vesselli, E.; de Gironcoli, S.; Lizzit, S.; Petaccia, L.; Zampieri, G.; Comelli, G.; Rosei, R. Highly Under-Coordinated Atoms at Rh Surfaces: Interplay of Strain and Coordination Effects on Core Level Shift. New J. Phys. 2007, 9, 143.

41. Baraldi, A.; Lizzit, S.; Comelli, G.; Kiskinova, M.; Rosei, R.; Honkala, K.; Nørskov, J. K. Spectroscopic Link between Adsorption Site Occupation and Local Surface Chemical Reactivity. Phys. Rev. Lett. 2004, 93, 046101.

42. Gustafson, J.; Mikkelsen, A.; Borg, M.; Andersen, J.; Lundgren, E.; Klein, C.; Hofer, W.; Schmid, M.; Varga, P.; Köhler, L.; et al. Structure of a Thin Oxide Film on Rh(100). Phys. Rev. B 2005, 71, 115442.

43. Bianchi, M.; Cassese, D.; Cavallin, A.; Comin, R.; Orlando, F.; Postregna, L.; Golfetto, E.; Lizzit, S.; Baraldi, A. Surface Core Level Shifts of Clean and Oxygen Covered Ir(111). New J. Phys. 2009, 11, 063002.

44. Hammer, B.; Nørskov, J. K. Why Gold Is the Noblest of All the Metals. Nature 1995, 376, 238-240.

45. Hammer, B.; Morikawa, Y.; Nørskov, J. K. CO Chemisorption at Metal Surfaces and Overlayers. Phys. Rev. Lett. 1996, 76, 2141-2144.

46. Cox, A. J.; Louderback, J. G.; Apsel, S. E.; Bloomfield, L. A. Magnetism in 4d-Transition Metal Clusters. Phys. Rev. B 1994, 49, 12295-12298. 
47. Ewers, T. D.; Sra, A. K.; Norris, B. C.; Cable, R. E.; Cheng, C.-H.; Shantz, D. F.; Schaak, R. E. Spontaneous Hierarchical Assembly of Rhodium Nanoparticles into Spherical Aggregates and Superlattices. Chem. Mater. 2005, 17, 514520.

48. Kresse, G.; Furthmüller, J. Efficient Iterative Schemes for $A b$ Initio Total-Energy Calculations Using a Plane-Wave Basis Set. Phys. Rev. B 1996, 54, 11169.

49. Perdew, J. P.; Burke, K.; Ernzerhof, M. Generalized Gradient Approximation Made Simple. Phys. Rev. Lett. 1996, 77, 3865.

50. Alfè, D. Ab Initio Molecular Dynamics, a Simple Algorithm for Charge Extrapolation. Comput. Phys. Commun. 1999, 118, 31.

51. Blöchl, P. E. Projector Augmented-Wave Method. Phys. Rev. B 1994, 50, 17953.

52. Kresse, G.; Joubert, D. From Ultrasoft Pseudopotentials to the Projector Augmented-Wave Method. Phys. Rev. B 1999, 59, 1758. 


\title{
Supporting Information
}

\section{Local Electronic Structure and Density of Edge and Facet Atoms at Rh Nanoclusters Self-Assembled on a Graphene Template}

\author{
Alberto Cavallin, ${ }^{\dagger, \ddagger}$ Monica Pozzo, ${ }^{\mathbb{I}}$ Cristina Africh, ${ }^{\S}$ Alessandro Baraldi, ${ }^{,+\dagger, \S}$ Erik \\ Vesselli, ${ }^{\dagger, \S}$ Carlo Dri, ${ }^{\S}$ Giovanni Comelli, ${ }^{\dagger}{ }^{\S}$ Rosanna Larciprete, $\|$ Paolo Lacovig, ${ }^{\perp}$ \\ Silvano Lizzit, ${ }^{\perp}$ and Dario Alfèd,\#\#
}

Physics Department and CENMAT, University of Trieste, Via Valerio 2, I-34127 Trieste, ITALY, Institute of Condensed Matter Physics, Ecole Polytechnique Fédérale de Lausanne, Station 3, CH-1015 Lausanne, SWITZERLAND, Department of Earth Sciences, Department of Physics and Astronomy, and London Centre for Nanotechnology, University College London, Gower Street, London WCIE 6BT, UNITED KINGDOM, IOM-CNR Laboratorio TASC, Area Science Park, S.S. 14 Km 163.5, I-34149 Trieste, ITALY, CNR-Institute for Complex Systems, Via Fosso del Cavaliere 100, 00133 Roma, ITALY, Sincrotrone Trieste S.C.p.A., Area Science Park, S.S. 14 Km 163.5, I-34149 Trieste, ITALY, and IOM-CNR, DEMOCRITOS National Simulation Centre, I-34100 Trieste, ITALY

E-mail: alessandro.baraldi@elettra.trieste.it

\footnotetext{
${ }^{*}$ To whom correspondence should be addressed

${ }^{\dagger}$ Physics Dep. and CENMAT, University of Trieste

†CMP, EPFL

IDep. of Earth Sciences, Dep. of Physics and Astronomy, and London Centre for Nanotechnology, UCL

§IOM-CNR Laboratorio TASC

CNR-Institute for Complex Systems

${ }^{\perp}$ Sincrotrone Trieste

\#IOM-CNR, DEMOCRITOS
} 


\section{Cluster model}

STM imaging of Rh nanoclusters obtained with $1 \mathrm{ML}$ deposition and annealed to $840 \mathrm{~K}$ exhibit a hexagonal shape and a well defined discrete height distribution (see Fig. 2f). Experimental line profiles across STM images reveal the presence of a flat top surface and of constant slope lateral nano-facets. Under particular tip conditions obtained only in a few images, the cluster lateral profile slope presents a change (see Fig. 1b of this Supporting Information). Even accounting for these slope changes, the STM images never display terraces parallel to the graphene surface. A possible reason for this fact is that the STM tip radius is typically much larger than the width of such terraces, i.e. the clusters do not present terraces parallel to the graphene surface wider than a few $\AA$. This is the condition for the occurrence of nano-facets almost as perfect as $\{111\}$ and $\{100\}$. The tentative structural model depicted below (Fig. 1a of this Supporting Information) was conceived based on the exposed considerations and on previous observations. ${ }^{1}$
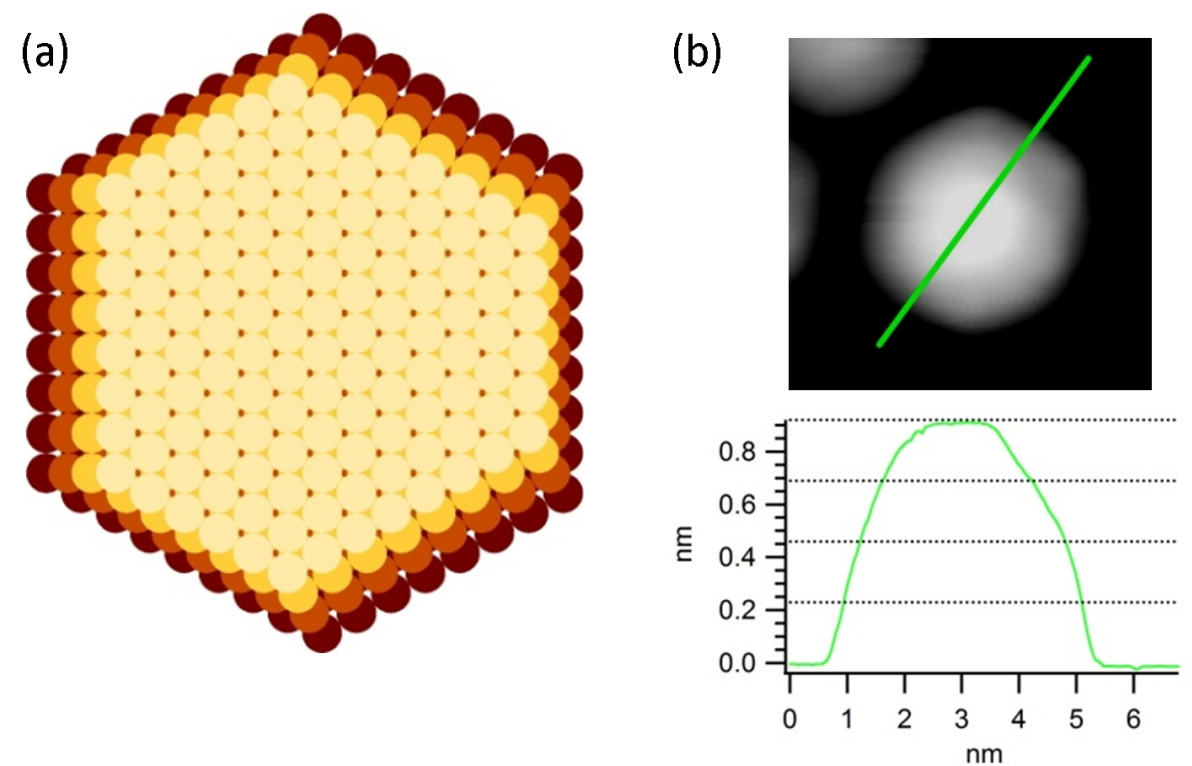

Figure 1: Cluster model based on STM images. (a) Model structure for a 4 layers cluster. Light yellow, yellow, orange and brown circles are top, intermediate (2 layers) and interface layer, resp. (b) Experimental line profile (bottom) across a cluster (top) obtained with particular tip conditions, exhibiting curvature changes at height levels corresponding to atomic Rh layers (dotted lines). Tunneling parameters: $\mathrm{I}=0.5 \mathrm{nA}, \mathrm{VB}=+0.5 \mathrm{~V}$. 


\section{Statistical Analysis of Nanocluster Morphological Properties: com- parison between STM and XPS information}

In order to further enquire on the $\mathrm{Rh}$ nanoclusters morphological properties and to relate them to the XPS coordination-dependent information we have performed a statistical analysis. We focused on the nanoclusters obtained after annealing to $840 \mathrm{~K}$ (Figure $1 \mathrm{f}$ in the manuscript), where the height histrogram shows three sharp peaks corresponding to 3, 4 and 5 atomic layers.

The height is a fundamental morphological parameter which, together with the nanocluster diameter, determines the number of atoms at the edges, at facets and in the bulk of the nanoclusters. Our data, presented in Figure 2 of this Supporting Information, clearly show that the most representative class of clusters prepared in these conditions is 4 layers high. The nanocluster diameters, measured at half-height, are in the $37 \pm 3 \AA$ range.

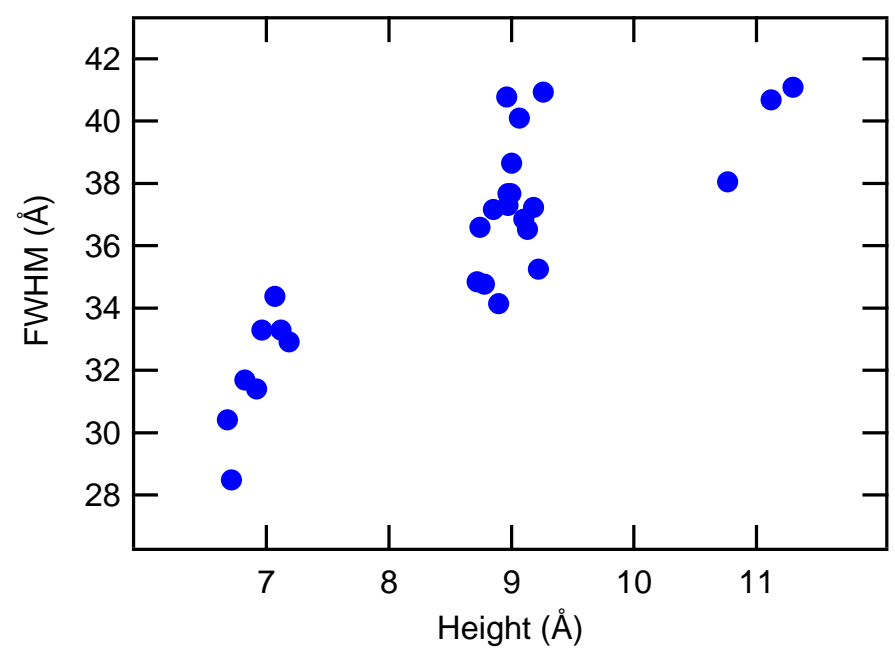

Figure 2: Single cluster Full Widths at Half Maximum (FWHM) and cluster Heights, obtained from STM images line profiles analysis.

It should be noted that STM obtained nanocluster Full Widths at Half Maximum (FWHM) are biased by the finite tip radius effect and therefore appear larger than the actual cluster diameter at half height. The cluster models we have analyzed present therefore diameters at half height, $d$, in the $33 \AA$ to $37 \AA$ range. 
The ball model cluster, reported in Figure 2 of the main manuscript and reproduced here in Figure 1a, is formed by 634 atoms on 4 layers and with $d=35 \AA$ it represents a realistic structural model of the most representative class of $\mathrm{Rh}$ nanoclusters.

In order to understand if this structural model is compatible also with XPS measurements we have computed the fractional intensity of the edge $\mathrm{Rh}_{E}$, facet $\mathrm{Rh}_{S}$ and bulk $\mathrm{Rh}_{B}$ core-level photoemission components for the spectrum obtained in similar conditions $\left(\theta=0.37 \mathrm{ML}, T_{\text {ann }}=\right.$ $840 \mathrm{~K}$, shown in Figure $3 \mathrm{~b}$ of the manuscript). We obtain $\mathrm{Rh}_{E}=15 \%, \mathrm{Rh}_{S}=53 \%, \mathrm{Rh}_{B}=32 \%$. These values compare to the fractional amounts for the model clusters, which are presented in Table 1.

Table 1: Fractional amounts of edge $\mathrm{Rh}_{E}$, facet $\mathrm{Rh}_{S}$ and bulk $\mathrm{Rh}_{B}$ atoms in 4 layers high model clusters of growing size, with half height diameters, $d$, going from $33 \AA$ to $37 \AA$.

\begin{tabular}{cccc}
$d$ & $\mathrm{Rh}_{E}$ & $\mathrm{Rh}_{S}$ & $\mathrm{Rh}_{B}$ \\
\hline $33 \AA$ & $16 \%$ & $48 \%$ & $36 \%$ \\
$35 \AA$ & $15 \%$ & $48 \%$ & $37 \%$ \\
$37 \AA$ & $14 \%$ & $48 \%$ & $38 \%$
\end{tabular}

Besides the very good agreement between the XPS and the STM obtained fractional amounts, the minor discrepancies can be explained as due to: (i) inelastic mean free path effects and photoelectron diffraction effects, which are always present in XPS analysis, (ii) the presence of clusters formed not only by 4 layers, but also by 3 and 5 layer, (iii) different initial Rh coverage in XPS and STM measurements.

\section{References}

1. Henry, C. R. Morphology of Supported Nanoparticles. Progr. Surf. Sci. 2005, 80, 92-116. 